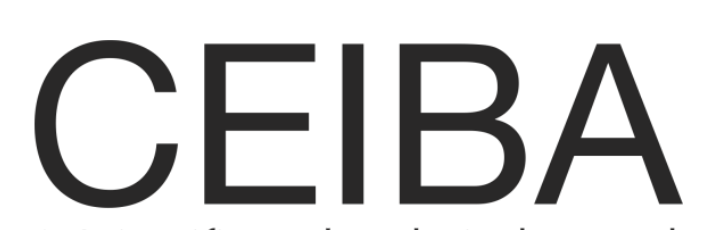

A Scientific and Technical Journal

Published by Zamorano

\title{
0846
}

\section{Módulos dinámicos de la madera de 17 especies determinados por vibraciones longitudinales}

\author{
Javier Ramón Sotomayor Castellanos \\ Facultad de Ingeniería en Tecnología de la Madera \\ Universidad Michoacana de San Nicolás de Hidalgo \\ Morelia, México

\section{Víctor López Garza} \\ Facultad de Ingeniería Mecánica \\ Universidad Michoacana de San Nicolás de Hidalgo \\ Morelia, México
}




\title{
Javier Ramón Sotomayor Castellanos y Víctor López Garza
}

Módulos dinámicos de la madera de 17 especies determinados por vibraciones longitudinales

Ceiba 0846: 1-11

DOI: $10.5377 /$ ceiba.v0i0846.8822

\author{
Publicado en el 2020 por \\ Escuela Agrícola Panamericana, Zamorano \\ P.O. Box 93 \\ Km 30 Tegucigalpa a Danlí \\ San Antonio de Oriente, Francisco Morazán, Honduras
}

Ceiba es la revista científica y tecnológica de la Escuela Agrícola Panamericana, Zamorano, publicada desde 1950. A partir del 2019, Ceiba se publica exclusivamente de manera electrónica y en el formato de publicación continua; esto con el propósito de acortar el tiempo entre aceptación de un artículo y su publicación, y que los autores puedan diseminar rápidamente los resultados de su investigación. Aceptamos artículos en español o en inglés relacionados con todas las áreas de la agricultura: agricultura tropical, recursos naturales, botánica, biología, ecología, desarrollo rural, forestales, economía agrícola, agronegocios, descripciones de nuevas especies, agroindustria, protección vegetal, y notas científicas (plagas nuevas, observaciones interesantes etc.). Ocasionalmente se publicarán revisiones bibliográficas y resúmenes de conferencias.

Los manuscritos sometidos tienen que ser originales y no deben haber sido publicados ni sometidos a otras revistas. Una vez sometidos a Ceiba tampoco deben someterse a otras revistas. Los autores deben indicar en su primer envío del artículo que cumplen con esta norma. Los artículos serán arbitrados por dos expertos antes de aceptarlos para publicación usando el sistema de doble ciego. Ceiba usa revisores externos, el autor puede sugerir los revisores, pero no se garantiza que se usen. Después de ser revisado, los comentarios de los revisores se regresan al autor con el cual se trabaja en el manuscrito si este fue aceptado. Si el artículo es aceptado, se deben hacer las correcciones sugeridas por los revisores y regresarlo al editor en no más de dos semanas.

Los autores deben enviar sus artículos a través del sitio web único de la revista: https://www.lamjol.info/index.php/CEIBA, para lo cual deben crear una cuenta en el mismo. Ceiba no dispone de ningún otro lugar para la recepción de manuscritos. En caso de algún problema, los autores pueden enviar por correo electrónico todos los archivos que forman el manuscrito (cuadros, fotografías, dibujos, y gráficas) al editor, Dr. Jesús Orozco, al correo electrónico jorozco@zamorano.edu. Adicionalmente, pueden comunicarse al teléfono +504 2287-2000 ext 2340.

Ceiba está indexada en Web of Science, latindex y Central American Journals Online (CAMJOL).

Editor: Jesús Orozco

Diagramación: Jesús Orozco y Katerin Aguilar

Comité editorial: Ronald D Cave, Mike D Owen, José Manuel Mora, Carlos Hernández DíazAmbrona, y Abelino Pitty

ISSN 2225-6687

Copyright. Este es un artículo de acceso abierto distribuido bajo los términos de Creative Commons, licencia de atribución no comercial, el cual permite el uso, distribución y reproducción no comercial en cualquier medio sin restricciones, siempre y cuando el autor original y la fuente sean acreditados. https://creativecommons.org/licenses/by-nc/3.0/deed.es. El copyright pertenece a los autores. 


\title{
Módulos dinámicos de la madera de 17 especies determinados por vibraciones longitudinales
}

\author{
Javier Ramón Sotomayor Castellanos \\ madera999@yahoo.com \\ Facultad de Ingeniería en Tecnología de la Madera \\ Universidad Michoacana de San Nicolás de Hidalgo \\ Morelia, México \\ Víctor López Garza \\ nepesh@hotmail.com \\ Facultad de Ingeniería Mecánica \\ Universidad Michoacana de San Nicolás de Hidalgo \\ Morelia, México
}

\begin{abstract}
Resumen. Los objetivos de la investigación fueron determinar el módulo de elasticidad dinámico por vibraciones longitudinales de 17 maderas, verificar los resultados experimentales con estimaciones empleando el método del elemento finito y contrastar los resultados con datos publicados. La hipótesis de trabajo propone que a medida que la densidad aparente de una madera aumenta, la magnitud de su módulo dinámico es mayor. Se realizaron pruebas de vibraciones longitudinales con 35 probetas de pequeñas dimensiones de 17 tipos de madera y se efectuaron modelados con el método del elemento finito. Las variables de respuesta fueron densidad aparente, frecuencia experimental y numérica, y el módulo dinámico. No se encontraron diferencias estadísticamente significativas entre las frecuencias experimentales y las numéricas. La densidad aparente correlaciona bien con el módulo dinámico. La magnitud del módulo dinámico de las 17 maderas estudiadas es similar al de las especies con densidades aparentes parecidas y reportadas para otras maderas. Los resultados son válidos para modelado con probetas de pequeñas dimensiones y en pruebas de vibraciones longitudinales descritos en esta investigación. Para el caso de estudios con piezas de madera de grandes dimensiones, se recomienda realizar estudios específicos.
\end{abstract}

Palabras clave: método del elemento finito, vibraciones longitudinales, frecuencia de vibración.

\section{Dynamic modules of the wood of 17 species determined by longitudinal vibrations}

\begin{abstract}
The objectives of the research were to determine the dynamic elastic modulus by longitudinal vibrations of 17 woods, to verify the experimental results with estimations using the finite element method and to contrast the results with published data. The working hypothesis proposes that as the apparent density of a wood increases, the magnitude of its dynamic modulus is greater. Longitudinal vibration tests were carried out with 35 small specimens of 17 types of wood and modeling was performed using the finite element method. The response variables were bulk density, experimental and numerical frequency, and dynamic modulus. No statistically significant differences were found between the experimental and the numerical frequencies. Bulk density correlates well with dynamic modulus. The magnitude of the dynamic modulus of the 17 woods studied is similar to that of the species with similar apparent densities and reported for other woods. The results are valid for modeling with small-dimension specimens and in longitudinal vibration tests described in this investigation. In the case of studies with large pieces of wood, it is recommended to carry out specific studies.
\end{abstract}

Key words: finite element method, longitudinal vibrations, vibration frequency.

\section{Introducción}

La biodiversidad de México y Centroamérica comprende una gran variedad de especies de madera (Cordero y Boshier 2003). Muchas de ellas tienen un bajo aprovechamiento en la industria de la madera, debido entre otros factores, al desconocimiento de sus características mecánicas, como lo es su módulo dinámico. La literatura reporta módulos dinámicos determinados por ultrasonido, ondas de esfuerzo y vibraciones transversales (Sotomayor Castellanos 2015); sin embargo, no se encontró información sobre pruebas de vibraciones longitudinales en especies de esta región. 
El módulo dinámico longitudinal de la madera, determinado en pruebas empleando probetas de pequeñas dimensiones, se utiliza principalmente para caracterizar especies de madera y de esta forma promocionar su uso en productos de valor agregado (Baar et al. 2015, Chauhan y Sethy 2016, Sotelo Montes et al. 2017). Sin embargo, para su correcta integración en el proyecto ingenieril o de arquitectura, es recomendable que los datos del módulo dinámico provengan de experimentos normalizados, y si es posible, trabajar con especímenes de tamaño similar al empleado en condiciones reales de servicio, como, por ejemplo, vigas y columnas (Arriaga et al. 2012, Cavalli et al. 2017).

Desde otra perspectiva, Baar et al. (2015), Chauhan y Sethy (2016) y Sotelo Montes et al. (2017), reportan datos del módulo dinámico de la madera determinado con vibraciones longitudinales y empleando probetas de pequeñas dimensiones. Así, los resultados derivados de pruebas con probetas de pequeñas dimensiones son válidos como referencia, y con los ajustes apropiados, pueden ser empleados como parámetros de ingeniería (Ilic 2003).

Uno de los paradigmas actuales en ciencias, ingeniería y tecnología de la madera es que la densidad aparente de la madera es la característica física de la madera empleada para predecir el módulo de elasticidad (Niklas y Spatz 2010). De igual modo, Sotomayor Castellanos y Correa Jurado (2016) proponen que: "Es necesario caracterizar el comportamiento mecánico de la madera con un enfoque de experimentación de caso por caso de una especie en particular. Cada procedimiento debe estar referido a las variables de referencia de las condiciones de ensayo, por ejemplo, la densidad aparente y el contenido de humedad de la madera, y con datos derivados de un tamaño de muestra observada estadísticamente representativa. Una vez teniendo observaciones integrantes y estadísticamente representativas, se pueden proponer tendencias en el comportamiento general para una especie en específico y/o por agrupamiento de varias de ellas que denoten una tendencia similar".

Los argumentos anteriores sostienen la hipótesis de que a medida que la densidad aparente de una especie de madera aumenta, la magnitud de su módulo dinámico será mayor.

El primer objetivo de esta investigación fue determinar el módulo de elasticidad dinámico por vibraciones longitudinales de 17 maderas endémicas de México y Centroamérica. La lista de especies y los códigos empleados se presentan en la Tabla 1. Por otra parte, las pruebas de vibraciones longitudinales de probetas de madera de pequeñas dimensiones están documentadas en Sotomayor Castellanos (2015). Debido a que no se detectaron trabajos realizados en las especies utilizadas en este estudio, ni de verificación de los resultados por medio de un procedimiento numérico, el segundo objetivo fue verificar los resultados experimentales contrastándoles con las estimaciones realizadas con el método del elemento finito. Un último objetivo fue contrastar los resultados con datos publicados.

\section{Materiales y métodos}

El estudio se llevó a cabo entre los meses de octubre y diciembre del 2018. Se recolectaron piezas de madera en aserraderos del Estado de Michoacán, México (19 $10^{\circ} 07^{\prime \prime} \mathrm{N}-101^{\circ} 53^{\prime} 59^{\prime \prime} \mathrm{O}$ ), de las 17 especies presentadas en la Tabla 1 . De cada especie se recortaron 35 probetas siguiendo las recomendaciones de la norma ISO 3129:2012 (International Organization for Standardization, 2012), con dimensiones de 0,02 $\mathrm{m} \times$ $0,02 \mathrm{~m}$ de sección transversal y $0,40 \mathrm{~m}$ de longitud, orientadas respectivamente en las direcciones radial, tangencial y longitudinal. Todas las probetas se elaboraron con madera sana y se revisó que estuviesen libres de anomalías de crecimiento. Las probetas fueron almacenadas durante 24 meses en una cámara de acondicionamiento con una temperatura de $20^{\circ} \mathrm{C}\left( \pm 1^{\circ} \mathrm{C}\right)$ y una humedad relativa del aire de $65 \%( \pm 2 \%)$, hasta lograr un peso constante correspondiente a un contenido de humedad promedio para todas las maderas de 9,7\%, con un coeficiente de variación de 10,4\%.

La densidad aparente de la madera se determinó por la razón entre el peso y el volumen de la probeta al momento del ensayo con un grupo complementario de 35 probetas pequeñas y de acuerdo con la norma ISO 13061-2:2014 (International Organization for Standardization 2014a). El contenido de humedad de la madera se calculó por el método de diferencia de pesos húmedo y seco, según la norma ISO 13061-1:2014 
(International Organization for Standardization 2014b). Las dimensiones de estas probetas fueron de 0,02 $\mathrm{m} \times 0,02 \mathrm{~m}$ de sección transversal por 0,06 m de longitud.

Las pruebas de vibraciones longitudinales consistieron en proporcionar un impacto en un extremo de la probeta orientado hacia su dirección longitudinal (Figura 1), siguiendo el procedimiento de Yoshihara (2012). La probeta estuvo apoyada sobre un soporte elástico en medio de la portada, correspondiente al nodo de su primer modo de vibración. Empleando el medidor de frecuencias Grindosonic ${ }^{\circledR}$ se midió la frecuencia natural del sistema en vibración definida como frecuencia experimental. Para esto, se capturó la señal de vibración por medio del micrófono del medidor, posicionado en el extremo opuesto al del impacto. Se realizaron tres repeticiones y su promedio se capturó como valor característico de una prueba.

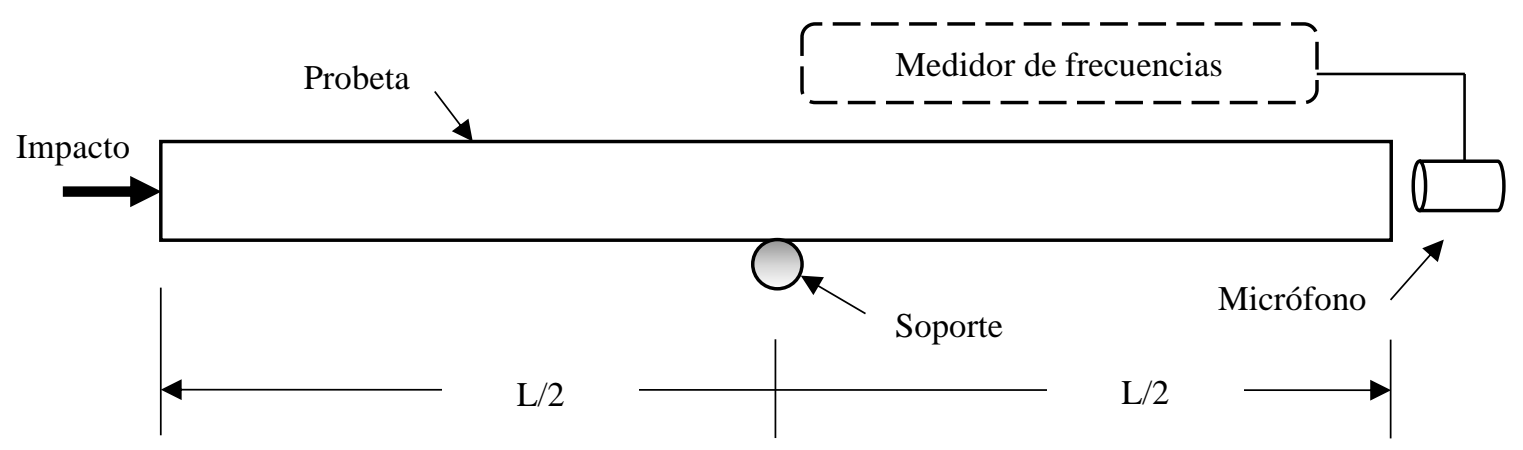

Figura 1. Esquema de las pruebas de vibraciones longitudinales. L = Longitud de la probeta.

El módulo dinámico se calculó con la ecuación de Yoshihara (2012) [1]:

$$
\mathrm{E}_{\mathrm{vl}}=4 \mathrm{~L}^{2} \rho_{\mathrm{CH}} \mathrm{f}_{\mathrm{exp}}^{2}
$$

Donde:

$\mathrm{E}_{\mathrm{vl}}=$ Módulo dinámico en vibraciones longitudinales $\left(\mathrm{MN} \mathrm{m}^{-2}\right)$

$\mathrm{L}=$ Longitud de la probeta $(\mathrm{m})$

$\rho \mathrm{cH}=$ Densidad aparente para un contenido de humedad $\mathrm{CH}\left(\mathrm{kg} \mathrm{m}^{-3}\right)$

$\mathrm{f}_{\mathrm{exp}}=$ Frecuencia experimental $(\mathrm{Hz})$

\section{Método del elemento finito}

La frecuencia teórica se estimó por medio del método del elemento finito (mef) siguiendo la metodología de Sotomayor Castellanos y López Garza (2016). La madera se modeló como un sólido elástico, macroscópicamente homogéneo, con propiedades de un medio continuo e isotrópico. Las probetas se idealizaron como vigas continuas, de geometría uniforme y de masa estructuralmente homogénea. Para cada una de las 17 especies se corrió un análisis modal con la herramienta parametrización del programa ANSYS $^{\circledR} 15$ (ANSYS Inc.). Para ello se utilizaron los valores experimentales de la densidad aparente y del módulo dinámico determinados previamente y se determinaron las frecuencias naturales del modelo, las cuales se definieron como las frecuencias numéricas $\left(f_{\text {mef }}\right)$.

Los principales parámetros que se utilizaron en el modelado fueron: tipo de elemento: Hexaédrico SOLID186; tamaño de la malla: 0,002 (m); número de elementos: 21100; número de nodos: 93293; función de forma: lineal (Figura 2a). El modelo de una probeta en su primer modo de vibración se presenta en la Figura 2b.

\section{Diseño experimental}

La unidad experimental consistió en 17 grupos de 35 probetas (Tabla 1). Las variables experimentales de respuesta fueron: densidad aparente $(\rho \mathrm{cH})$, frecuencia experimental (fexp), y módulo dinámico $\left(\mathrm{E}_{\mathrm{vl}}\right)$. La frecuencia estimada por el método del elemento finito $\left(f_{\mathrm{mef}}\right)$ se consideró variable numérica de respuesta. 

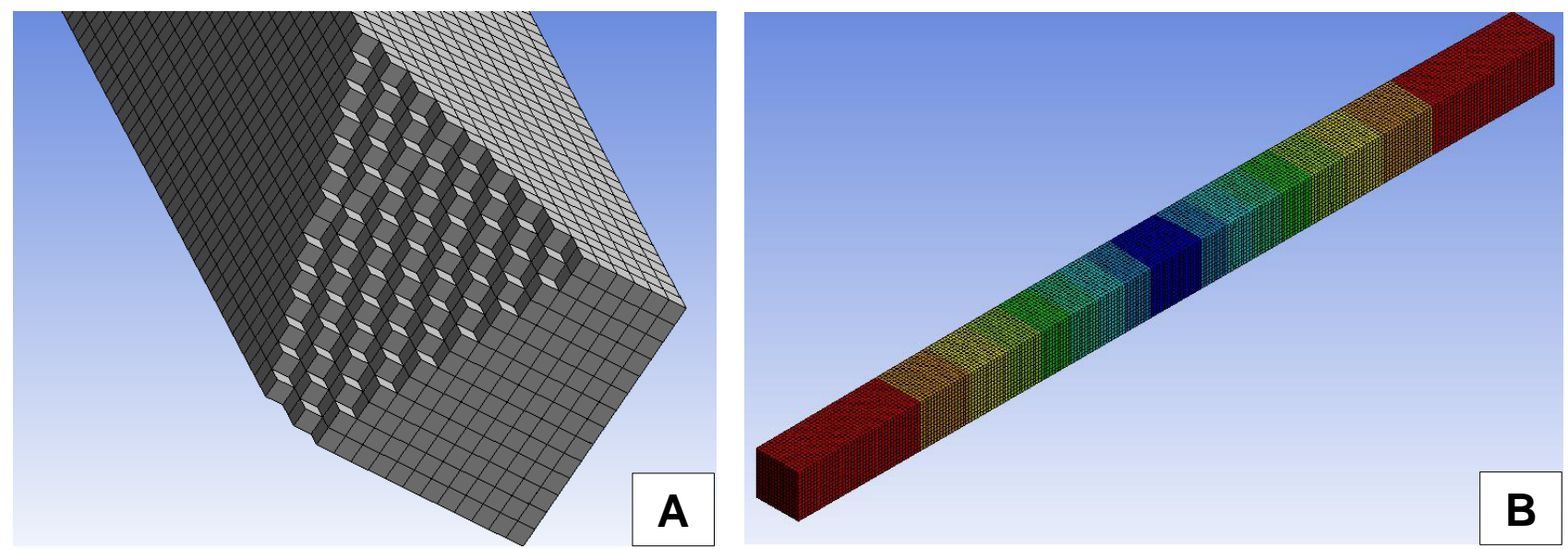

Figura 2. Modelo de las probetas usadas. A) Detalle del mallado. B) Modelo de una probeta.

Para cada variable experimental se calcularon su media $(\overline{\mathrm{x}})$ su desviación estándar $(\sigma)$, y su coeficiente de variación porcentual $(\mathrm{CV}=\sigma / \overline{\mathrm{x}})$. Para la variable numérica se calculó su media $(\overline{\mathrm{x}})$.

Las frecuencias experimental y numérica se consideraron muestras independientes. La estimación por el método del elemento finito se consideró el factor de variación. Para estas dos variables se determinó su normalidad en la distribución con el criterio de demarcación para el sesgo estandarizado (SE), y para el apuntamiento estandarizado (AE): [-2 < SE y/o $\mathrm{AE}<+2]$; se verificó la igualdad de varianzas (Ver-var) con la hipótesis: $\mathrm{H}_{0}: \sigma_{\mathrm{vl}}=\sigma_{\text {mef }}$ con un nivel de significancia de $5 \%(\alpha=0,05)$.

Los subíndices vl y mef se refieren a las muestras experimentales y numéricas respectivamente. Posteriormente se practicó un análisis de varianza (Anova) con la hipótesis nula: $\mathrm{H}_{0}: \overline{\mathrm{x}}_{\mathrm{vl}}=\overline{\mathrm{x}}_{\text {mef }}$ con un nivel de significancia de $5 \%(\alpha=0,05)$. El criterio de demarcación fue aceptar una diferencia estadísticamente significativa para valores $\mathrm{P}<0,05$.

Utilizando los resultados, se calculó la regresión lineal $(\mathrm{y}=\mathrm{ax}+\mathrm{b})$ y su coeficiente de determinación $\left(\mathrm{R}^{2}\right)$ entre la frecuencia numérica $\left(f_{\mathrm{mef}}\right)$ y la experimental $\left(f_{\exp }\right)$. Además, se calculó la correlación entre el módulo dinámico $\left(\mathrm{E}_{\mathrm{vl}}\right)$ y la densidad aparente $(\rho \mathrm{cH})$. Estos últimos resultados se contrastaron con Baar et al. (2015), Chauhan y Sethy (2016) y Sotelo Montes et al. (2017).

\section{Resultados y discusión}

Los resultados del promedio de la densidad aparente, frecuencia experimental y numérica y del módulo dinámico, así como su respectivo coeficiente de variación, son presentados para las 17 especies en la Tabla 1. Las especies están ordenadas en relación ascendente a su densidad aparente.

La Tabla 2 presenta los datos de la densidad aparente y de los módulos dinámicos, así como sus coeficientes de variación, de 18 especies reportadas por Baar et al. (2015) con un contenido de humedad de $8 \%$, Chauhan y Sethy (2016) con un contenido de humedad de 12\%, y Sotelo Montes et al. (2017) con un contenido de humedad de $11 \%$.

\section{Análisis estadístico}

Los valores promedio determinados experimental y numéricamente provienen de distribuciones normales $\left(\mathrm{P}_{(\mathrm{a}=0,05)}<0,05 ; \mathrm{f}_{\text {exp }}\right.$ : $\mathrm{SE}=-0,0638$ y AE $=0,3883 ; \mathrm{f}_{\text {mef: }} \mathrm{SE}=-0,1852$ y $\left.\mathrm{AE}=-0,2751\right)$. No existe una diferencia estadísticamente significativa entre las medias de las dos variables (f $f_{\exp } v s$. $\left.\mathrm{f}_{\mathrm{mef}}\right)\left(\mathrm{P}_{(\mathrm{a}=0,05)}\right.$ Ver-vara $=0,9735$ y $\mathrm{P}_{(\alpha=0,05)}$ Anova $\left.=0,7669\right)$. Estos corolarios permiten verificar los resultados experimentales empleando el modelado por el método del elemento finito. 


\section{Densidad aparente}

La densidad aparente determinada comprendió el rango de valores promedio que va desde $351 \mathrm{~kg} \mathrm{~m}^{-3}$ (Spathodea campanulata) hasta $996 \mathrm{~kg} \mathrm{~m}^{-3}$ (Cordia elaeagnoides). Paralelamente, el coeficiente de variación de la densidad aparente, al interior de una especie, fue mínimo para Fraxinus uhdei (4,8\%) y máximo para Cupressus lindleyi (19,5\%). Estos valores de densidad aparente y su coeficiente de variabilidad son comparables con las reportadas para las mismas especies por Tamarit Urias y López Torres (2007), Silva Guzmán et al. (2010) y Sotomayor Castellanos (2015). La Figura 3 muestra las dispersiones de los coeficientes de variación de las densidades aparentes (CV $\left.\rho_{\mathrm{CH}}\right)$ en función de las densidades aparentes $\left(\rho_{\mathrm{CH}}\right)$ de esta investigación y de Baar et al. (2015), Chauhan y Sethy (2016) y Sotelo Montes et al. (2017).

No obstante que los coeficientes de variación se sobreponen en la misma nube de datos, se observan diferencias entre especies. Esta diversidad puede explicarse por la variabilidad en la magnitud de las características físicas que existe entre ellas y al interior de una especie (Perré et al. 2016). La diversidad en las propiedades físico-mecánicas de la madera también depende de factores genéticos y ambientales y se encuentra en todos los niveles entre gimnospermas y angiospermas, árboles de climas tropicales y templados, poblaciones, individuos, y ubicaciones de muestreo dentro del árbol (Zhang et al. 2011).

Tabla 1. Densidad aparente $\left(\rho_{\mathrm{cH}}\right)$, frecuencia experimental ( $\left.f_{\mathrm{exp}}\right)$, frecuencia numérica ( $\left.f_{\mathrm{mef}}\right)$, y módulo dinámico $\left(\mathrm{E}_{\mathrm{vl}}\right)$ de 17 especies de madera.

\begin{tabular}{|c|c|c|c|c|}
\hline Especie & $\begin{array}{c}\rho \mathrm{CH} \\
\left(\mathrm{kg} \mathrm{m}^{-3}\right)\end{array}$ & $\begin{array}{c}f_{\exp } \\
(\mathrm{Hz})\end{array}$ & $\begin{array}{c}\mathbf{f}_{\mathrm{fem}} \\
(\mathrm{Hz})\end{array}$ & $\begin{array}{c}\mathbf{E}_{\mathrm{vl}} \\
\left(\mathrm{MN} \mathrm{\textrm {m } ^ { - 2 }}\right)\end{array}$ \\
\hline Spathodea campanulata & $\begin{array}{c}351 \\
(6,6)^{*}\end{array}$ & $\begin{array}{c}3498 \\
(35,4)\end{array}$ & $\begin{array}{c}3498 \\
-\end{array}$ & $\begin{array}{c}3081 \\
(35,7)\end{array}$ \\
\hline Enterolobium cyclocarpum & $\begin{array}{c}456 \\
(10,4)\end{array}$ & $\begin{array}{l}4561 \\
(6,6)\end{array}$ & $\begin{array}{c}4526 \\
-\end{array}$ & $\begin{array}{c}5907 \\
(12,6)\end{array}$ \\
\hline Cedrela odorata & $\begin{array}{c}462 \\
(12,1)\end{array}$ & $\begin{array}{c}5966 \\
(13,2)\end{array}$ & $\begin{array}{c}6025 \\
-\end{array}$ & $\begin{array}{l}10604 \\
(25,8)\end{array}$ \\
\hline Cupressus lindleyi & $\begin{array}{c}473 \\
(19,5)\end{array}$ & $\begin{array}{l}5148 \\
(9,6)\end{array}$ & $\begin{array}{c}5210 \\
-\end{array}$ & $\begin{array}{l}8119 \\
(36,6)\end{array}$ \\
\hline Swietenia macrophylla & $\begin{array}{c}540 \\
(6,7)\end{array}$ & $\begin{array}{l}5358 \\
(9,5)\end{array}$ & $\begin{array}{c}5401 \\
-\end{array}$ & $\begin{array}{c}9960 \\
(17,1)\end{array}$ \\
\hline Tabebuia donnell-smithii & $\begin{array}{c}602 \\
(7,1)\end{array}$ & $\begin{array}{l}4944 \\
(5,4)\end{array}$ & $\begin{array}{c}4986 \\
-\end{array}$ & $\begin{array}{c}9462 \\
(13,9)\end{array}$ \\
\hline Fraxinus uhdei & $\begin{array}{c}611 \\
(4,8)\end{array}$ & $\begin{array}{c}5157 \\
(12,0)\end{array}$ & $\begin{array}{c}5231 \\
-\end{array}$ & $\begin{array}{l}10571 \\
(23,5)\end{array}$ \\
\hline Tabebuia rosea & $\begin{array}{c}613 \\
(9,6)\end{array}$ & $\begin{array}{l}5732 \\
(5,4)\end{array}$ & $\begin{array}{c}5763 \\
-\end{array}$ & $\begin{array}{l}12873 \\
(11,1)\end{array}$ \\
\hline Fagus mexicana & $\begin{array}{c}621 \\
(5,2)\end{array}$ & $\begin{array}{l}5375 \\
(4,8)\end{array}$ & $\begin{array}{c}5420 \\
-\end{array}$ & $\begin{array}{l}11535 \\
(12,6)\end{array}$ \\
\hline Fraxinus americana & $\begin{array}{c}652 \\
(5,6)\end{array}$ & $\begin{array}{l}5613 \\
(9,1)\end{array}$ & $\begin{array}{c}5659 \\
-\end{array}$ & $\begin{array}{l}13202 \\
(16,0)\end{array}$ \\
\hline Dalbergia palo-escrito & $\begin{array}{c}662 \\
(10,2)\end{array}$ & $\begin{array}{c}5600 \\
(12,2)\end{array}$ & $\begin{array}{c}5694 \\
-\end{array}$ & $\begin{array}{l}13571 \\
(29,6)\end{array}$ \\
\hline Guazuma ulmifolia & $\begin{array}{c}675 \\
(6,0)\end{array}$ & $\begin{array}{c}4621 \\
(14,4)\end{array}$ & $\begin{array}{c}4703 \\
-\end{array}$ & $\begin{array}{c}9437 \\
(30,3)\end{array}$ \\
\hline Andira inermis & $\begin{array}{c}737 \\
(5,0)\end{array}$ & $\begin{array}{l}4827 \\
(8,0)\end{array}$ & $\begin{array}{c}4877 \\
-\end{array}$ & $\begin{array}{l}11083 \\
(16,9)\end{array}$ \\
\hline Juglans pyriformis & $\begin{array}{r}773 \\
(7,7)\end{array}$ & $\begin{array}{l}5273 \\
(6,9)\end{array}$ & $\begin{array}{c}5327 \\
-\end{array}$ & $\begin{array}{l}13868 \\
(17,8)\end{array}$ \\
\hline Albizia plurijuga & $\begin{array}{l}838 \\
(3,5)\end{array}$ & $\begin{array}{l}5747 \\
(9,0)\end{array}$ & $\begin{array}{c}5809 \\
-\end{array}$ & $\begin{array}{l}17879 \\
(18,5)\end{array}$ \\
\hline Quercus sp. & $\begin{array}{c}866 \\
(6,3)\end{array}$ & $\begin{array}{l}6223 \\
(6,0)\end{array}$ & $\begin{array}{c}6274 \\
-\end{array}$ & $\begin{array}{l}21551 \\
(13,9)\end{array}$ \\
\hline Cordia elaeagnoides & $\begin{array}{c}996 \\
(9,7)\end{array}$ & $\begin{array}{l}5455 \\
(6,4)\end{array}$ & $\begin{array}{c}5491 \\
-\end{array}$ & $\begin{array}{l}18985 \\
(14,5)\end{array}$ \\
\hline
\end{tabular}




\section{Frecuencia experimental}

La frecuencia medida en cada probeta, idealizada esta como un sistema en movimiento en su primer modo de vibración, depende de su módulo dinámico, densidad aparente, geometría, y localización de los apoyos del sistema (Yoshihara, 2012). En la presente investigación, las dimensiones y el soporte de las probetas fueron similares en todas las pruebas y para todas las especies. Sin embargo, la densidad aparente, expresada como la relación peso/volumen, fue particular a cada probeta. De aquí, se puede comparar la frecuencia entre especies de esta investigación, pero no es posible hacerlo con los resultados de otros autores, los cuales utilizaron dimensiones y configuraciones de ensayo diferentes.

Tabla 2. Datos reportados por Baar et al. (2015), Chauhan y Sethy (2016), y Sotelo Montes et al. (2017).

\begin{tabular}{|c|c|c|c|}
\hline Especie & Referencia & $\begin{array}{c}\rho \mathrm{\rho CH} \\
\left(\mathrm{kg} \mathrm{m}^{-3}\right)\end{array}$ & 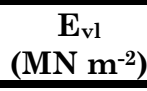 \\
\hline Afzelia bipindensis & Baar et al. (2015) & $\begin{array}{c}766 \\
(6,2)^{*}\end{array}$ & $\begin{array}{l}12860 \\
(18,2)\end{array}$ \\
\hline Intsia bijuga & Baar et al. (2015) & $\begin{array}{c}812 \\
(9,1)\end{array}$ & $\begin{array}{l}18220 \\
(13,4)\end{array}$ \\
\hline Millettia laurentii & Baar et al. (2015) & $\begin{array}{c}840 \\
(10,1)\end{array}$ & $\begin{array}{l}17920 \\
(11,9)\end{array}$ \\
\hline Astronium graveolens & Baar et al. (2015) & $\begin{array}{c}856 \\
(10,2)\end{array}$ & $\begin{array}{l}16570 \\
(15,0)\end{array}$ \\
\hline Microberlinia brazzavillensis & Baar et al. (2015) & $\begin{array}{c}810 \\
(6,6)\end{array}$ & $\begin{array}{l}19620 \\
(14,6)\end{array}$ \\
\hline Maesopsis eminii & Chauhan y Sethy (2016) & $\begin{array}{c}357 \\
(6,8)\end{array}$ & $\begin{array}{l}9250 \\
(9,7)\end{array}$ \\
\hline Bombax ceiba & Chauhan y Sethy (2016) & $\begin{array}{c}437 \\
(9,2)\end{array}$ & $\begin{array}{c}9160 \\
(15,8)\end{array}$ \\
\hline Melia dubia & Chauhan y Sethy (2016) & $\begin{array}{c}577 \\
(11,6)\end{array}$ & $\begin{array}{c}13640 \\
(7,3)\end{array}$ \\
\hline Grevillea robusta & Chauhan y Sethy (2016) & $\begin{array}{c}614 \\
(4,6)\end{array}$ & $\begin{array}{c}10610 \\
(9,8)\end{array}$ \\
\hline Hevea brasiliensis & Chauhan y Sethy (2016) & $\begin{array}{c}621 \\
(3,9)\end{array}$ & $\begin{array}{l}9360 \\
(9,6)\end{array}$ \\
\hline Tectona grandis & Chauhan y Sethy (2016) & $\begin{array}{c}632 \\
(7,0)\end{array}$ & $\begin{array}{l}14700 \\
(13,3)\end{array}$ \\
\hline Acacia auriculiformis & Chauhan y Sethy (2016) & $\begin{array}{c}722 \\
(7,8)\end{array}$ & $\begin{array}{c}16770 \\
(9,9)\end{array}$ \\
\hline Eucalyptus (híbrido) & Chauhan y Sethy (2016) & $\begin{array}{c}726 \\
(3,8)\end{array}$ & $\begin{array}{l}17310 \\
(12,5)\end{array}$ \\
\hline Balanites aegyptiaca & Sotelo Montes et al. (2017) & $\begin{array}{r}730 \\
(6,2)\end{array}$ & $\begin{array}{l}14545 \\
(21,7)\end{array}$ \\
\hline Combretum glutinosum & Sotelo Montes et al. (2017) & $\begin{array}{c}798 \\
(8,2)\end{array}$ & $\begin{array}{l}18947 \\
(20,6)\end{array}$ \\
\hline Guiera senegalensis & Sotelo Montes et al. (2017) & $\begin{array}{l}808 \\
(6,3)\end{array}$ & $\begin{array}{l}17489 \\
(20,5)\end{array}$ \\
\hline Piliostigma reticulatum & Sotelo Montes et al. (2017) & $\begin{array}{c}656 \\
(9,6)\end{array}$ & $\begin{array}{l}14106 \\
(22,5)\end{array}$ \\
\hline Ziziphus mauritiana & Sotelo Montes et al. (2017) & $\begin{array}{c}693 \\
(9,1)\end{array}$ & $\begin{array}{l}15287 \\
(21,7)\end{array}$ \\
\hline
\end{tabular}

$\rho \mathrm{cH}=$ Densidad aparente; $\mathrm{E}_{\mathrm{vl}}=$ Módulo dinámico; * $(\mathrm{CV})=$ Coeficiente de variación en porciento .

La magnitud de la frecuencia experimental (Tabla 1), fluctuó entre $3498 \mathrm{~Hz}$ para S. campanulata y un $6223 \mathrm{~Hz}$ correspondiente a Quercus sp., y no presentó relación alguna con la densidad aparente de la especie $\left(f_{\exp }=-2,3475 \rho \mathrm{CH}+12833, \mathrm{R}^{2}=0,0055\right)$. El coeficiente de variación de la frecuencia varió entre 4,8\% (Fagus mexicana) y $35,4 \%$ (S. campanulata) y la diferencia promedio entre las frecuencias experimentales y numéricas fue de $-0,92 \mathrm{~Hz}$. El análisis de correlación lineal entre las frecuencias experimental $\left(f_{\exp }\right)$ y 
numérica $\left(f_{\text {mef }}\right)$, realizado con datos de la Tabla 1 , resultó en un coeficiente de determinación $\left(\mathrm{R}^{2}\right)$ de 0,99 (Figura 4). Este corolario verifica los resultados experimentales de esta investigación al contrastarlos con las estimaciones numéricas con el método del elemento finito.

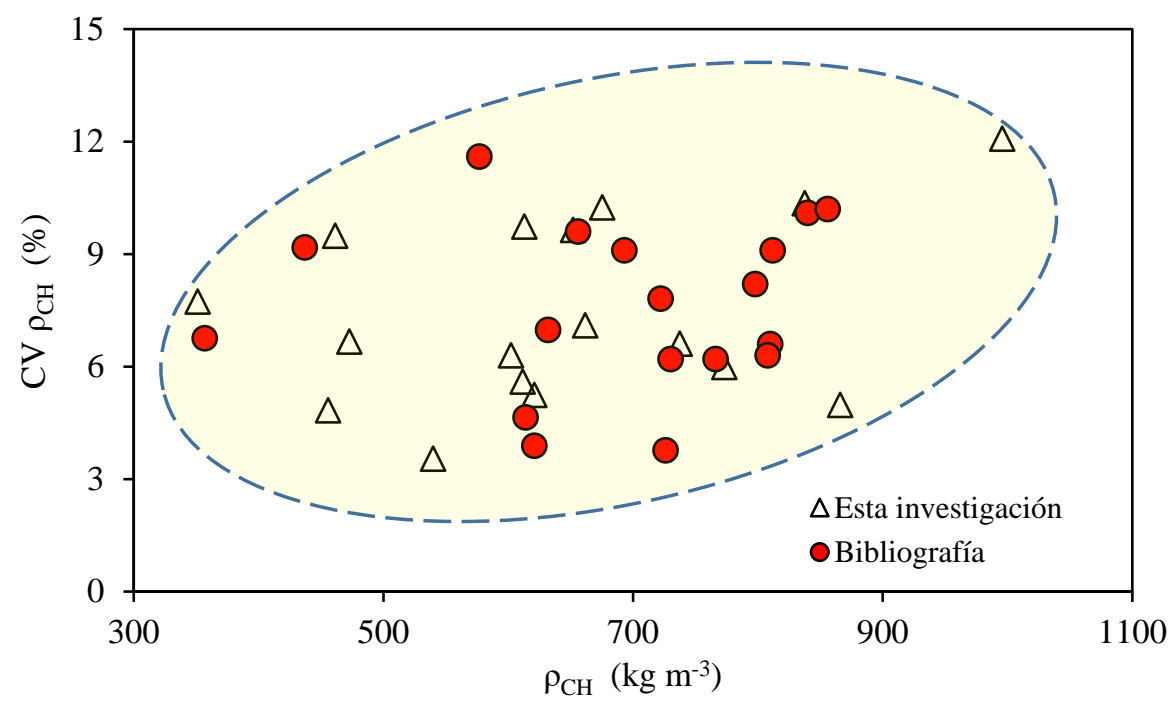

Figura 3. Dispersiones de los coeficientes de variación de las densidades aparentes, (CV $\left.\rho_{\mathrm{CH}}\right)$ en función de las densidades aparentes $(\rho \mathrm{cH})$ de esta investigación y de Baar et al. (2015), Chauhan y Sethy (2016) y Sotelo Montes et al. (2017).

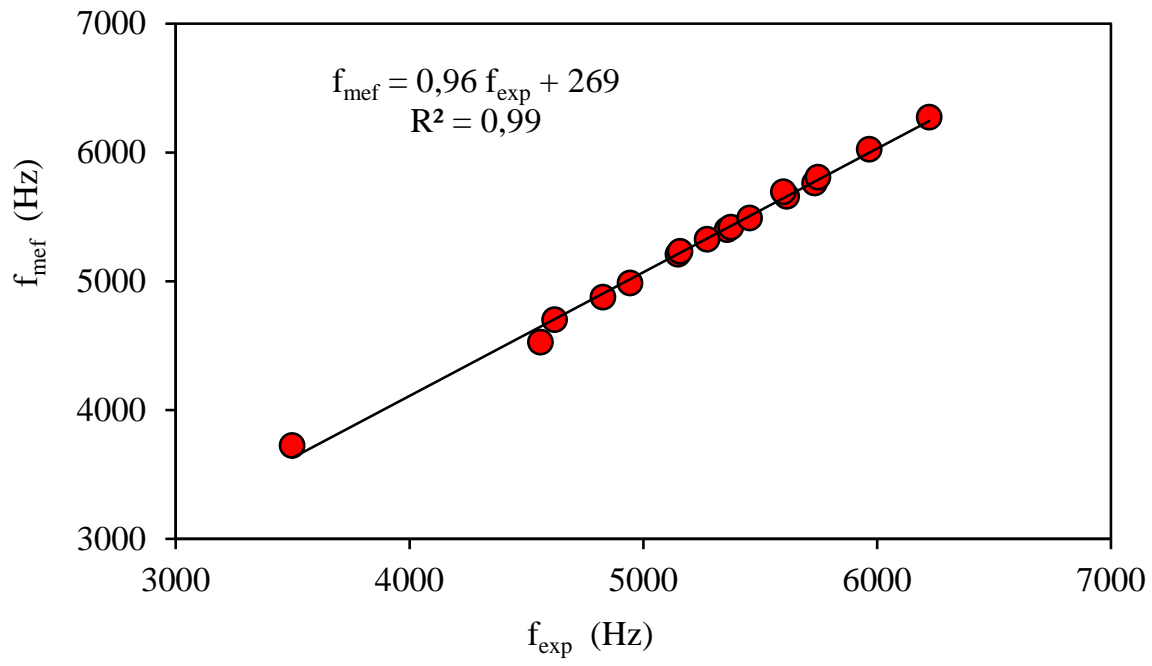

Figura 4. Correlación y su coeficiente de determinación $\left(\mathrm{R}^{2}\right)$ entre las frecuencias numéricas ( $\mathrm{f}_{\text {mef }}$ y las frecuencias experimentales $\left(f_{\text {exp }}\right)$.

\section{Módulo dinámico}

El módulo dinámico mínimo correspondió a $S$. campanulata $\left(3081 \mathrm{MN} \mathrm{m}^{-2}\right)$ y el máximo fue para $C$. elaeagnoides (18985 $\mathrm{MN} \mathrm{m}^{-2}$ ). El coeficiente de variación osciló entre 11,1\% (Tabebuia rosea) y 65,7\% (S. campanulata). Los coeficientes de variación de los módulos dinámicos de 11 de las 17 maderas fueron de misma magnitud que los publicados (Tabla 1 y Figura 5). Las diferencias se encontraron en: S. campanulata, Cedrela odorata, C. lindleyi, F. uhdei, Dalbergia palo-escrito, y Guazuma ulmifolia, cuyos coeficientes fueron superiores a 23,5\%. Este resultado confirma para las 17 maderas estudiadas, el paradigma aceptado en 
Ciencias, Ingeniería y Tecnología de la madera el cual sugiere que la resistencia mecánica de la madera, en este caso representada por el módulo dinámico, aumenta proporcionalmente a su densidad aparente (Niklas y Spatz 2010).

La dispersión de los resultados del módulo dinámico de las 17 especies en proporción a su densidad aparente fue similar a la de las especies reportadas por Baar et al. (2015), Chauhan y Sethy (2016), y Sotelo Montes et al. (2017) (Figura 6). La magnitud del módulo dinámico aumentó con la densidad aparente con un fuerte coeficiente de determinación entre estas dos variables $\left(R^{2}=0,80\right)$. Este coeficiente fue superior en un $14,3 \%$ respecto al reportado por los autores citados que fue de $\mathrm{R}^{2}=0,70$. En este trabajo, el coeficiente de determinación indica únicamente una medida de la capacidad de la densidad aparente como parámetro predictor del módulo dinámico.

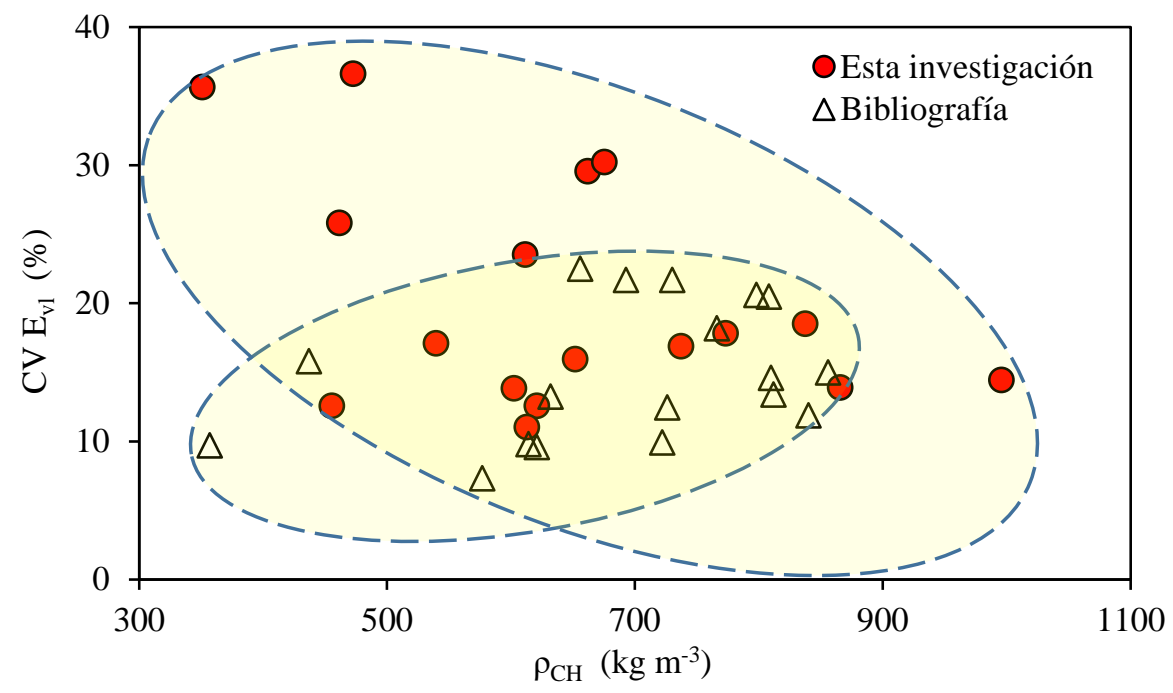

Figura 5. Dispersión de los coeficientes de variación de los módulos dinámicos $\left(\mathrm{CV} \mathrm{E}_{\mathrm{vl}}\right)$ en función de la densidad aparente $\left(\rho_{\mathrm{CH}}\right)$ y de Baar et al. (2015), Chauhan y Sethy (2016) y Sotelo Montes et al. (2017).

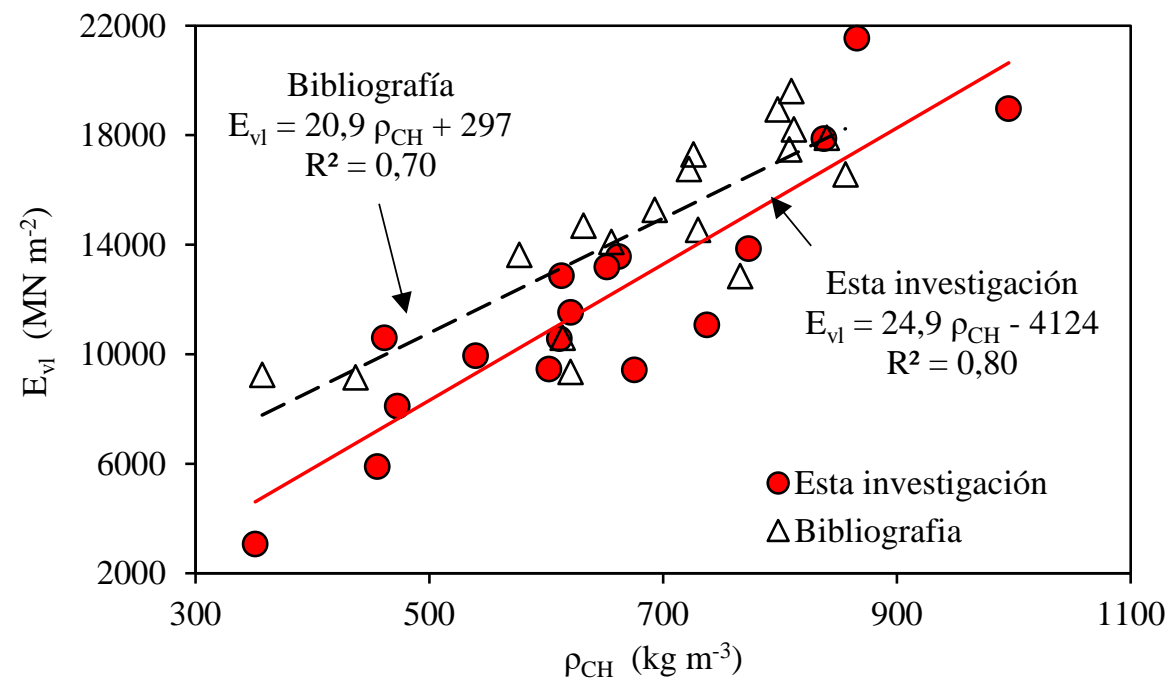

Figura 6. Regresiones lineales y sus coeficientes de determinación $\left(\mathrm{R}^{2}\right)$ de los módulos dinámicos ( $\left.\mathrm{E}_{\mathrm{vl}}\right)$ en función de las densidades aparentes $\left(\rho_{\mathrm{CH}}\right)$ para los resultados de esta investigación y para los datos de Baar et al. (2015), Chauhan y Sethy (2016) y Sotelo Montes et al. (2017). 


\section{Variabilidad de los resultados}

Las magnitudes del módulo dinámico de la madera obtenidas en esta investigación (Tabla 1) son muy diversas y son similares a las reportadas por los autores revisados (Tabla 2). Varias causas han sido sugeridas para esta variabilidad: la biodiversidad entre especies forestales (Brémaud et al. 2012), la heterogeneidad anatómica (Schubert et al. 2009), la anisotropía material (Brémaud et al. 2011), y la higroscopicidad del tejido leñoso (Mvondo et al. 2017). Una causa adicional que provoca la variabilidad del módulo dinámico de la madera son las diferentes configuraciones de las pruebas con que se miden los parámetros necesarios para calcular este parámetro (Bachtiar et al. 2017). Esta diversidad representa una ventaja de este material para fines de cálculo ingenieril y para el diseño de productos a base de madera; sin embargo, se requiere considerar los resultados especie por especie.

\section{Conclusiones}

Se determinó la densidad aparente de la madera y el módulo de elasticidad dinámico por vibraciones longitudinales de 17 especies endémicas de México y Centroamérica. La magnitud del módulo dinámico de las 17 maderas estudiadas es similar al de las especies con densidades aparentes parecidas y reportadas para otras maderas en la bibliografía.

Se confirmó que la densidad aparente de la madera es la característica física que puede predecir el módulo dinámico y que el modelado numérico por el método del elemento finito es útil para predecir y estimar el módulo dinámico longitudinal.

Los resultados son válidos para modelado con probetas de pequeñas dimensiones y en pruebas de vibraciones longitudinales descritos en esta investigación. Para el caso de estudio con piezas de madera de grandes dimensiones, se recomienda realizar estudios específicos y mejorar el modelo numérico.

\section{Literatura citada}

Arriaga F, Íñiguez-González G, Esteban M, Divos F. 2012. Vibration method for grading of large crosssection coniferous timber species. Holzforschung. 66(3):381-387.

ANSYS Inc. ANSYS Software version 15. Canonsburg, Pennsylvania, Estados Unidos.

Baar J, Tippner J, Rademacher P. 2015. Prediction of mechanical properties - modulus of rupture and modulus of elasticity - of five tropical species by nondestructive methods. Maderas: Ciencia y Tecnología. 17(2): 239-252.

Bachtiar EV, Sanabria SJ, Mittig JP, Niemz P. 2017. Moisture-dependent elastic characteristics of walnut and cherry wood by means of mechanical and ultrasonic test incorporating three different ultrasound data evaluation techniques. Wood Science and Technology. 51(1):47-67.

Brémaud I, Gril J, Thibaut B. 2011. Anisotropy of wood vibrational properties: dependence on grain angle and review of literature data. Wood Science and Technology. 45(4):735-754.

Brémaud I, El Kaïm Y, Guibal D, Minato K, Thibaut B, Gril J. 2012. Characterization and categorization of the diversity in viscoelastic vibrational properties between 98 wood types. Annals of Forest Science. 69(3):373-386.

Cavalli A, Marcon B, Cibecchini D, Mazzanti P, Fioravanti M, Procino L, Togni M. 2017. Dynamic excitation and FE analysis to assess the shear modulus of structural timber. Materials and Structures. 50(2):1-8. 
Chauhan S, Sethy A. 2016. Differences in dynamic modulus of elasticity determined by three vibration methods and their relationship with static modulus of elasticity. Maderas: Ciencia y Tecnología. 18(2):373-382.

Cordero J, Boshier DH. 2003. Árboles de Centroamérica. Turrialba: Centro Agronómico Tropical de Investigación y Enseñanza. 1079 p.

Ilic J. 2003. Dynamic MOE of 55 species using small wood beams. HolzalsRoh- und Werkstoff. 61(3):167172.

International Organization for Standardization. 2012. ISO 3129:2012. Wood. Sampling methods and general requirements for physical and mechanical testing of small clear wood specimens. Ginebra: International Organization for Standardization. 9p.

International Organization for Standardization. 2014a. ISO 13061-2:2014. Wood. Determination of density for physical and mechanical tests. Ginebra: International Organization for Standardization. 9 p.

International Organization for Standardization. 2014b. ISO 13061-1:2014. Wood. Determination of moisture content for physical and mechanical tests. Ginebra: International Organization for Standardization. 6 p.

Mvondo RRN, Meukam P, Jeong J, Meneses DD, Nkeng EG. 2017. Influence of water content on the mechanical and chemical properties of tropical wood species. Results in Physics. 7:2096-2103.

Niklas KJ, Spatz HC. 2010. Worldwide correlations of mechanical properties and green wood density. American Journal of Botany. 97(10):1587-1594.

Perré P, Almeida G, Ayouz M, Frank X. 2016. New modelling approaches to predict wood properties from its cellular structure: image-based representation and meshless methods. Annals of Forest Science. 73(1):147-162.

Schubert S, Gsell D, Dual J, Motavalli M, Niemz P. 2009. Acoustic wood tomography on trees and the challenge of wood heterogeneity. Holzforschung. 63(1):107-112.

Silva Guzmán JA, Fuentes Talavera FJ, Rodríguez Anda R, Torres Andrade PA, Lomelí Ramírez MA, Ramos Quirarte J, Waitkus C, Richter HG. 2010. Fichas de propiedades tecnológicas y usos de maderas nativas de México e importadas. México: Comisión Nacional Forestal. 124 p.

Sotelo Montes C, Weber JC, Garcia Rosilei A, Silva Dimas A, Muñiz GIB. 2017. Variation in growth, wood stiffness and density, and correlations between growth and wood stiffness and density in five tree and shrub species in the Sahelian and Sudanian ecozones of Mali. Trees. 31(3):833-849.

Sotomayor Castellanos JR. 2015. Banco FITECMA de características físico-mecánicas de maderas mexicanas. Morelia: Universidad Michoacana de San Nicolás de Hidalgo. 69 p.

Sotomayor Castellanos JR, López Garza V. 2016. Verificación por el método del elemento finito del comportamiento mecánico de la madera en flexión estática. Investigación e Ingeniería de la Madera. 12(3):4-55.

Sotomayor Castellanos JR, Correa Jurado S. 2016. Retención de sales de boro en la madera y su efecto en el módulo de elasticidad dinámico. Revista Científica. 24(1):67-76.

Tamarit Urias JC, López Torres JL. 2007. Xilotecnología de los principales árboles tropicales de México. México: Instituto Nacional de Investigaciones Forestales Agropecuarias y Pecuarias. 264 p. 
Sotomayor Castellanos y López Garza. Ceiba 2020, 0846: 1-11. Módulos dinámicos de la madera por vibraciones longitudinales

Yoshihara H. 2012. Examination of the specimen configuration and analysis method in the flexural and longitudinal vibration tests of solid wood and wood-based materials. Forest Products Journal. 62(3):191-200.

Zhang T, Bai SL, Bardet S, Alméras T, Thibaut B, Beauchêne J. 2011. Radial variations of vibrational properties of three tropical woods. Journal of Wood Science. 57(5):377-386.

Recibido noviembre 25, 2019; aceptado junio 28, 2020.

Cómo citar: Sotomayor Castellanos JR, López Garza V. 2020. Módulos dinámicos de la madera de 17 especies determinados por vibraciones longitudinales. Ceiba. 0846:1-11. 